\title{
Tuning of optical phonons by Fermi level in graphene
}

\author{
Tsuneya Ando \\ Department of Physics, Tokyo Institute of Technology \\ 2-12-1 Ookayama, Meguro-ku, Tokyo 152-8551, Japan
}

\begin{abstract}
A brief review is given on recent theoretical study on continuum models of optical phonons as well as zone-boundary phonons and effects of electron-phonon interaction in monolayer and bilayer graphene, resulting in strong dependence of the frequency and broadening on the electron density.
\end{abstract}

Keywords: electron-phonon interaction, magnetophonon resonance, phonon anomaly

\section{Introduction}

Recently, monolayer graphene was fabricated using the socalled scotch-tape technique [1] and the magnetotransport was measured including the integer quantum Hall effect [2, 3]. Since then the graphene became the subject of extensive theoretical and experimental study [4, 5]. The carbon nanotube is graphene rolled into a cylindrical form, discovered and synthesized earlier than graphene [6]. The purpose of this paper is to give a brief review on optical phonons as well as zoneboundary phonons and effects of electron-phonon interaction in monolayer and bilayer graphene and in nanotubes.

\section{Monolayer Graphene and Nanotube}

In a monolayer graphene the conduction and valence bands consisting of $\pi$ orbitals cross at $\mathrm{K}$ and $\mathrm{K}$ ' points of the Brillouin zone, where the Fermi level is located [7, 8]. Electronic states near a $\mathrm{K}$ point are described by a $\mathbf{k} \cdot \mathbf{p}$ equation equivalent to Weyl's equation for a neutrino or a Dirac equation with vanishing rest mass $[6,9,10,11,12,13,14]$. In the vicinity of the $\mathrm{K}$ point, in particular, we have

$$
\gamma(\vec{\sigma} \cdot \hat{\mathbf{k}}) \mathbf{F}(\mathbf{r})=\varepsilon \mathbf{F}(\mathbf{r}), \quad \mathbf{F}(\mathbf{r})=\left(\begin{array}{l}
F_{A}(\mathbf{r}) \\
F_{B}(\mathbf{r})
\end{array}\right),
$$

where $F_{A}$ and $F_{B}$ describe the amplitude at sublattice points $\mathrm{A}$ and $\mathrm{B}$, respectively, $\gamma$ is a band parameter, $\hat{\mathbf{k}}=\left(\hat{k}_{x}, \hat{k}_{y}\right)$ is the wave-vector operator, and $\vec{\sigma}=\left(\sigma_{x}, \sigma_{y}\right)$ is the Pauli matrix. The equation of motion for the $\mathrm{K}^{\prime}$ point is obtained by replacing $\vec{\sigma}$ with $\vec{\sigma}^{*}$ in the above equation.

Electronic states in a carbon nanotube $(\mathrm{CN})$ are obtained by imposing generalized periodic boundary condition $\mathbf{F}(\mathbf{r}+\mathbf{L})=$ $\exp (\mp 2 \pi v i / 3) \mathbf{F}(\mathbf{r})$ (upper sign for $\mathrm{K}$ and lower for $\mathrm{K}^{\prime}$ ) in the circumference direction specified by chiral vector $\mathbf{L}$ with $v=0$ or \pm 1 determined by the $\mathrm{CN}$ structure. We have $v=0$ for a metallic $\mathrm{CN}$ and $v= \pm 1$ for a semiconducting $\mathrm{CN}$.

\section{Long-Wavelength Optical Phonon}

Long-wavelength optical phonons are known to be measured directly by the Raman scattering $[15,16]$. Usually, they are described perfectly well in a continuum model. Such a model was developed and the Hamiltonian for electron-phonon interactions was derived [17], and effects of electron-phonon interaction on optical phonons were recently studied in graphene $[18,19]$ and in nanotubes $[17,20]$.

Optical phonons are represented by the relative displacement of two sub-lattice atoms A and B,

$$
\mathbf{u}(\mathbf{r})=\sum_{\mathbf{q}, \mu} \sqrt{\frac{\hbar}{2 N M \omega_{0}}}\left(b_{\mathbf{q} \mu}+b_{-\mathbf{q} \mu}^{\dagger}\right) \mathbf{e}_{\mu}(\mathbf{q}) e^{i \mathbf{q} \cdot \mathbf{r}},
$$

where $N$ is the number of unit cells, $M$ is the mass of a carbon atom, $\omega_{0}$ is the phonon frequency at the $\Gamma$ point, $\mathbf{q}=\left(q_{x}, q_{y}\right)$ is the wave vector, $\mu$ denotes the modes (' $\mathrm{t}$ ' for transverse and ' 1 ' for longitudinal), and $b_{\mathbf{q} \mu}^{\dagger}$ and $b_{\mathbf{q} \mu}$ are the creation and destruction operators, respectively. For $q_{x}=q \cos \varphi_{\mathbf{q}}$ and $q_{y}=q \sin \varphi_{\mathbf{q}}$ with $q=|\mathbf{q}|$, we have $\mathbf{e}_{1}(\mathbf{q})=i\left(\cos \varphi_{\mathbf{q}}, \sin \varphi_{\mathbf{q}}\right)$ and $\mathbf{e}_{\mathrm{t}}(\mathbf{q})=i\left(-\sin \varphi_{\mathbf{q}}, \cos \varphi_{\mathbf{q}}\right)$.

The interaction between optical phonons and an electron in the vicinity of the $\mathrm{K}$ and $\mathrm{K}$ ' points is given by [17]

$$
\mathcal{H}_{\text {int }}^{\mathrm{K}}=-\sqrt{2} \frac{\beta_{\Gamma} \gamma}{b^{2}} \vec{\sigma} \times \mathbf{u}(\mathbf{r})
$$

where $b$ is the bond length, $\beta_{\Gamma}=d \ln \gamma_{0}(b) / d \ln b$ with $\gamma_{0}$ being the the hopping integral in a nearest-neighbor tight-binding model, and vector product for vectors $\mathbf{p}=\left(p_{x}, p_{y}\right)$ and $\mathbf{q}=$ $\left(q_{x}, q_{y}\right)$ in two dimensions is defined by $\mathbf{p} \times \mathbf{q}=p_{x} q_{y}-p_{y} q_{x}$. We have $\beta_{\Gamma} \sim 2$ in conventional semiconductors [21] and $\beta_{\Gamma}$ may be slightly larger in carbon systems.

The lattice distortion gives rise to a shift in the origin of the wave vector or an effective vector potential, i.e., $u_{x}$ in the $y$ direction and $u_{y}$ in the $x$ direction. The Hamiltonian for the $\mathrm{K}^{\prime}$ point is obtained by replacing $\vec{\sigma}$ by $-\vec{\sigma}^{*}$. The interaction 


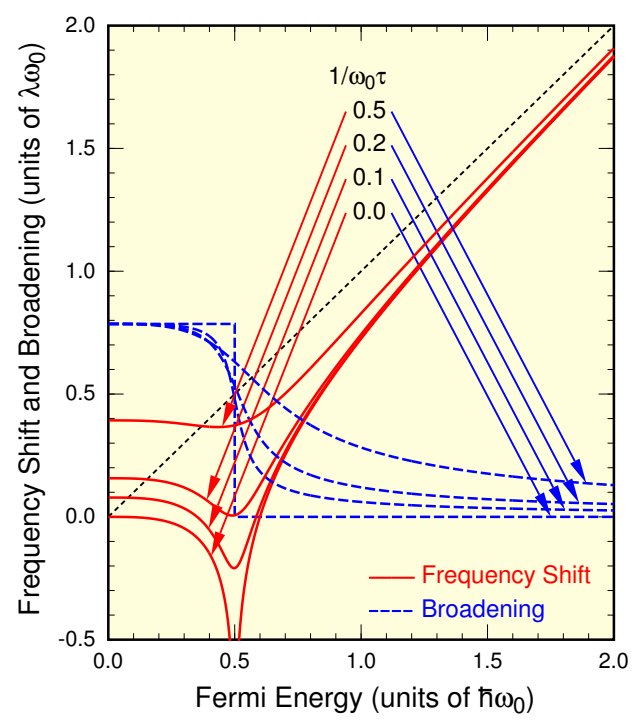

Figure 1: The frequency shift and broadening of optical phonons in monolayer graphene as a function of the Fermi energy. $\tau$ is a phenomenological relaxation time characterizing the level broadening effect due to disorder.

strength is characterized by the dimensionless coupling parameter

$$
\lambda_{\Gamma}=\frac{36 \sqrt{3}}{\pi} \frac{\hbar^{2}}{2 M a^{2}} \frac{1}{\hbar \omega_{0}}\left(\frac{\beta_{\Gamma}}{2}\right)^{2} .
$$

For $M=1.993 \times 10^{-23} \mathrm{~g}$ and $\hbar \omega_{0}=0.196 \mathrm{eV}$, we have $\lambda_{\Gamma} \approx 3 \times 10^{-3}\left(\beta_{\Gamma} / 2\right)^{2}$. This shows that the interaction is not strong and therefore the lowest order perturbation gives sufficiently accurate results.

The phonon Green's function is written as

$$
D_{\mu}(\mathbf{q}, \omega)=\frac{2 \hbar \omega_{0}}{(\hbar \omega)^{2}-\left(\hbar \omega_{0}\right)^{2}-2 \hbar \omega_{0} \Pi_{\mu}(\mathbf{q}, \omega)} .
$$

The phonon frequency is determined by the pole of $D_{\mu}(\mathbf{q}, \omega)$. In the case of weak interaction, the shift of the phonon frequency, $\Delta \omega_{\mu}$, and the broadening, $\Gamma_{\mu}$, are given by

$$
\Delta \omega_{\mu}=\frac{1}{\hbar} \operatorname{Re} \Pi_{\mu}\left(\mathbf{q}, \omega_{0}\right), \quad \Gamma_{\mu}=-\frac{1}{\hbar} \operatorname{Im} \Pi_{\mu}\left(\mathbf{q}, \omega_{0}\right) .
$$

When we calculate the self-energy of optical phonons starting with the known phonon modes in graphene, its direct evaluation causes a problem of double counting [22]. In fact, if we apply the above formula to the case of vanishing Fermi energy, we get the frequency shift due to virtual excitations of all electrons in the $\pi$ bands. However, this contribution is already included in the definition of the frequency $\omega_{0}$. In order to avoid such a problem, we have to subtract the contribution in the undoped graphene for $\omega=0$ corresponding to the adiabatic approximation.

Figure 1 shows the frequency shift and broadening for various values of $1 / \omega_{0} \tau$. For nonzero $\delta$ or $1 / \omega_{0} \tau$, the logarithmic singularity of the frequency shift and the sharp drop in the broadening at $\varepsilon_{\mathrm{F}}=\hbar \omega_{0} / 2$ disappear, but the corresponding features remain for $1 / \omega_{0} \tau \ll 1$, where $\varepsilon_{\mathrm{F}}$ is the Fermi energy. Similar results were reported independently [23] and experiments giving qualitatively similar results were reported [24, 25].
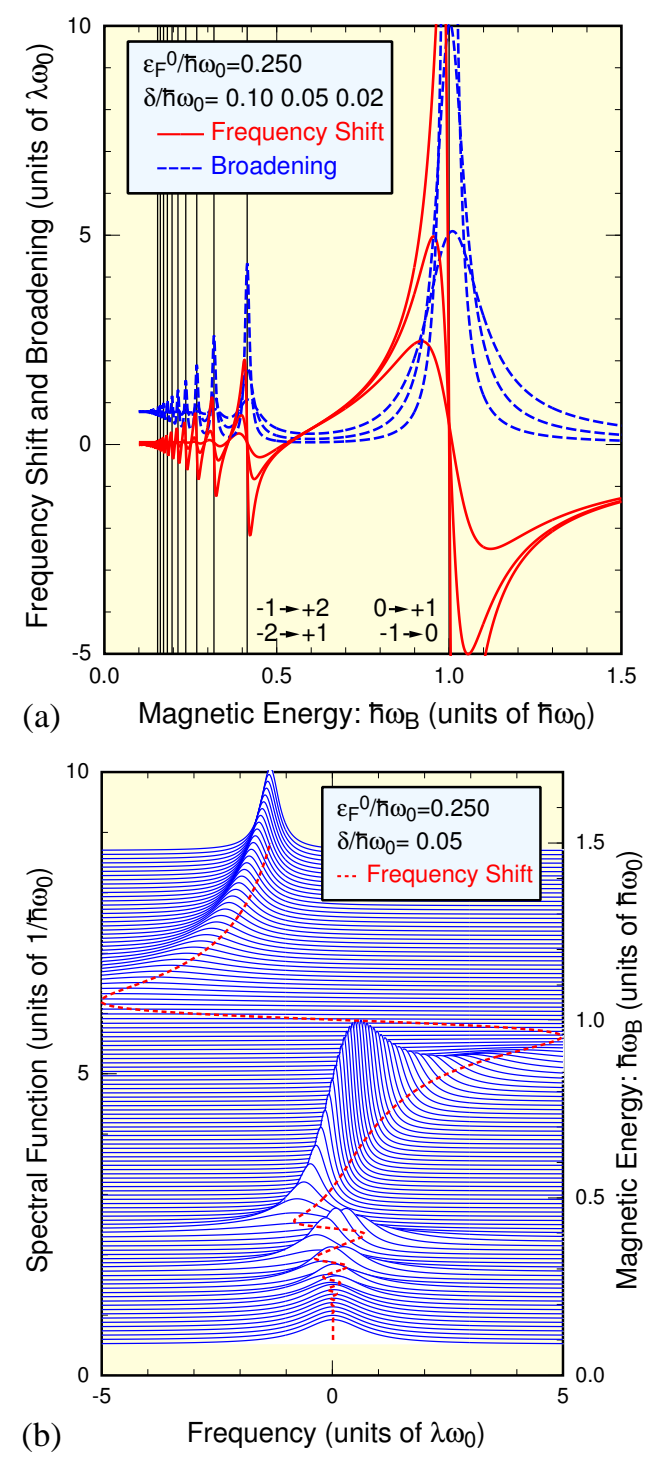

Figure 2: (a) The frequency shift and broadening of optical phonons in monolayer graphene as a function of effective magnetic energy $\hbar \omega_{B}$. Thin vertical lines show resonance magnetic fields. $\varepsilon_{\mathrm{F}}^{0} / \hbar \omega_{0}=0.25$. The results for $\delta / \hbar \omega_{0}=0.1,0.05$, and 0.02 are shown. (b) The phonon spectral function for $\delta / \hbar \omega_{0}=0.05$. The dotted line shows the peak position as a function of $\hbar \omega_{B}$.

The calculation can easily be extended to the case in the presence of magnetic field $B$, where discrete Landau levels are formed and oscillations due to resonant interactions appear in the frequency shift and the broadening [19]. The Landau-level energy is given by $\varepsilon_{n}=\operatorname{sgn}(n) \sqrt{|n|} \hbar \omega_{B}(n=0, \pm 1, \cdots)$, where $\operatorname{sgn}(n)$ denotes the sign of $n$ and $\hbar \omega_{B}=\sqrt{2} \gamma / l$ with magnetic length $l=\sqrt{c \hbar / e B}$. Figure 2 shows calculated frequency shift and broadening when $\varepsilon_{\mathrm{F}}^{0} / \hbar \omega_{0}=0.25$ and the corresponding phonon spectral function, where $\varepsilon_{\mathrm{F}}^{0}$ is the Fermi energy in the absence of a magnetic field. All resonant transitions from $-n$ to $n+1$ and from $-n-1$ to $n$ with $n>0$ appear at the field where their energy difference becomes equal to $\hbar \omega_{0}$. At resonances, the phonon spectrum exhibits characteristic behavior. This magneto-phonon resonance was studied also in Ref. [26] and recently observed in Raman experiments [27]. 

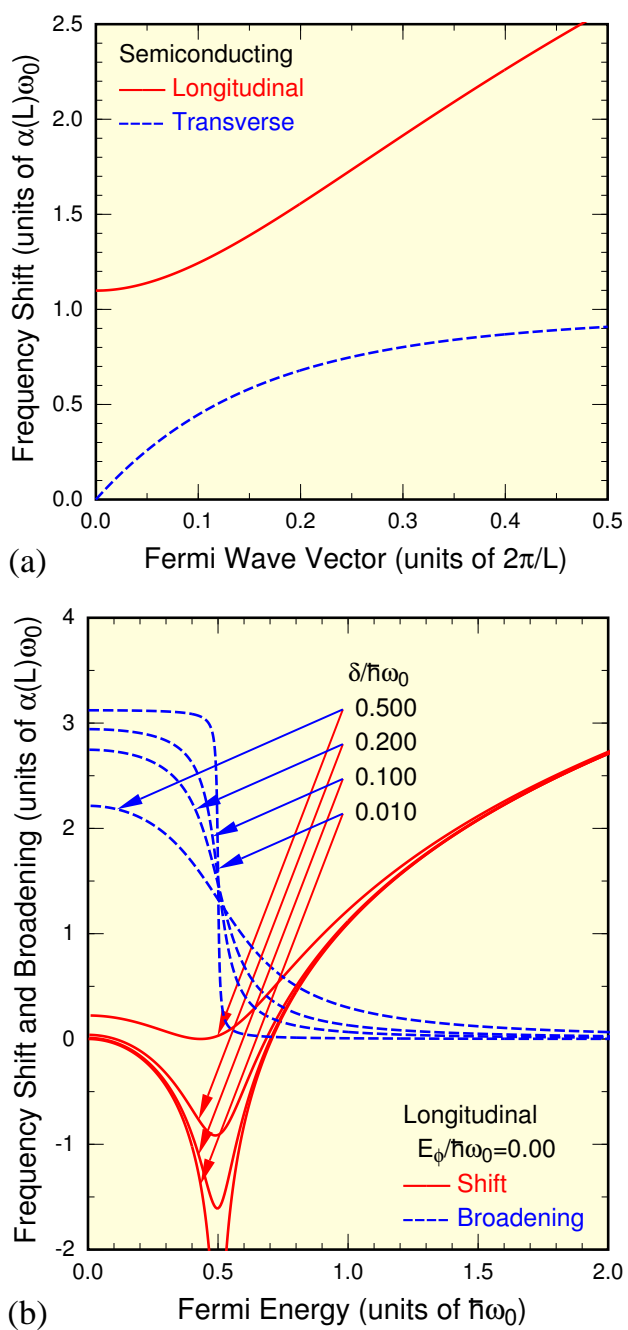

Figure 3: (a) The frequency shift in a semiconducting nanotube as a function of the Fermi wave vector $k_{\mathrm{F}}$. The solid and dashed lines represent the longitudinal and transverse modes, respectively. (b) The frequency shift and broadening of the longitudinal mode in metallic nanotubes as a function of the Fermi energy.

The same tuning of the optical-phonon frequency and broadening due to change in the Fermi level is also possible in carbon nanotubes. In fact, effects of the electron-phonon interaction on the optical phonon in carbon nanotubes were theoretically studied earlier than in graphene [17]. In nanotubes, the modes are classified into longitudinal and transverse, depending on their displacement in the axis or circumference direction. Figure 3 shows the results of the similar calculations in carbon nanotubes [20].

In semiconducting nanotubes, the imaginary part vanishes identically because of the presence of a gap. The frequency of the longitudinal and transverse modes is both shifted to higher frequency side and the shift is smaller for the longitudinal mode for small $k_{\mathrm{F}}$. The behavior of two modes as a function of $k_{\mathrm{F}}$ is similar to that of "level crossing." In metallic nanotubes, the transverse mode is not affected by the doping at all. For the longitudinal mode, the energy shift exhibits a downward shift and considerable broadening [17]. For nonzero $k_{\mathrm{F}}$, the self-energy has a logarithmic divergence at $\gamma k_{\mathrm{F}}=\hbar \omega_{0} / 2$ and increases log- arithmically with $k_{\mathrm{F}}$ for $\gamma k_{\mathrm{F}}>\hbar \omega_{0} / 2$ for vanishing $\delta$. This behavior in $\mathrm{CN}$, the same as in graphene theoretically predicted $[18,19,23,28]$ and experimentally observed [24, 25], was observed in Raman experiments [29, 30] and discussed theoretically $[31,32]$.

\section{Zone-Boundary Phonon}

Phonons near the $\mathrm{K}$ and $\mathrm{K}$ ' point, called zone-boundary phonons, can play important roles in intervalley scattering between the $\mathrm{K}$ and $\mathrm{K}$ ' points. In general, there exist four independent eigen modes for each wave vector. However, after straightforward calculations, we can see that only one mode with the highest frequency contributes to the electron-phonon interaction [33]. This mode is known as a Kekulé type distortion generating only bond-length changes. The interaction Hamiltonian is given by

$$
\mathcal{H}_{\text {int }}=2 \frac{\beta_{K} \gamma}{b^{2}}\left(\begin{array}{cc}
0 & \omega^{-1} \Delta(\mathbf{r}) \sigma_{y} \\
\omega \Delta^{\dagger}(\mathbf{r}) \sigma_{y} & 0
\end{array}\right),
$$

where $\omega=e^{2 \pi i / 3}$ and $\beta_{K}$ is another appropriate parameter, which is equal to $\beta_{\Gamma}$ for the tight-binding model. In the second quantized form,

$$
\Delta(\mathbf{r})=\sum_{\mathbf{q}} \sqrt{\frac{\hbar}{2 N M \omega_{K}}}\left(b_{K \mathbf{q}}+b_{K^{\prime}-\mathbf{q}}^{\dagger}\right) e^{i \mathbf{q} \cdot \mathbf{r}},
$$

where $\omega_{K}$ is the frequency of the Kekule mode. It is worth noting that $\Delta$ cannot be given by a simple summation over the $\mathrm{K}$ and $\mathrm{K}$ ' modes. We should take a proper linear combination of the $\mathrm{K}$ and $\mathrm{K}$ ' modes in order to make the lattice displacement a real variable. We can easily understand the operator form of $\Delta$ and $\Delta^{\dagger}$ in the interaction Hamiltonian by considering the momentum conservation with the fact that $2 \mathbf{K}-\mathbf{K}^{\prime}$ and $\mathbf{K}-2 \mathbf{K}^{\prime}$ are reciprocal lattice vectors, where $\mathbf{K}$ and $\mathbf{K}^{\prime}$ are the wave vectors at the $\mathrm{K}$ and $\mathrm{K}$ ' point. The dimensionless coupling parameter, $\lambda_{K}$, is given by the same expression as Eq. (4) except that $\omega_{0}$ is replaced with $\omega_{K}$ and $\beta_{\Gamma}$ with $\beta_{K}$. For $\hbar \omega_{K}=161.2 \mathrm{meV}$, we have $\lambda_{K}=3.5 \times 10^{-3}\left(\beta_{K} / 2\right)^{2}$.

The lifetime of an electron with energy $\varepsilon$ is given by the scattering probability from the initial state to possible final states via emission and absorption of one phonon. For the zone-center phonon, the summation of the contributions of longitudinal and transverse modes gives isotropic scattering probability in each of the $\mathrm{K}$ and $\mathrm{K}$ ' points. For the zone-boundary phonon, an electron around the K point can be scattered to the K' point accompanied by absorption of one phonon around the $\mathrm{K}$ point or by the emission of one phonon around the K' point. Further, backward scattering is stronger than forward scattering, suggesting that the zone-boundary phonon is more effective in reducing the electron velocity in high-field transport.

In graphene, the calculated scattering probabilities for both phonons are given by the same formula,

$$
\frac{\hbar}{\tau}=\pi \lambda_{\alpha}\left|\varepsilon-\hbar \omega_{\alpha}\right|
$$




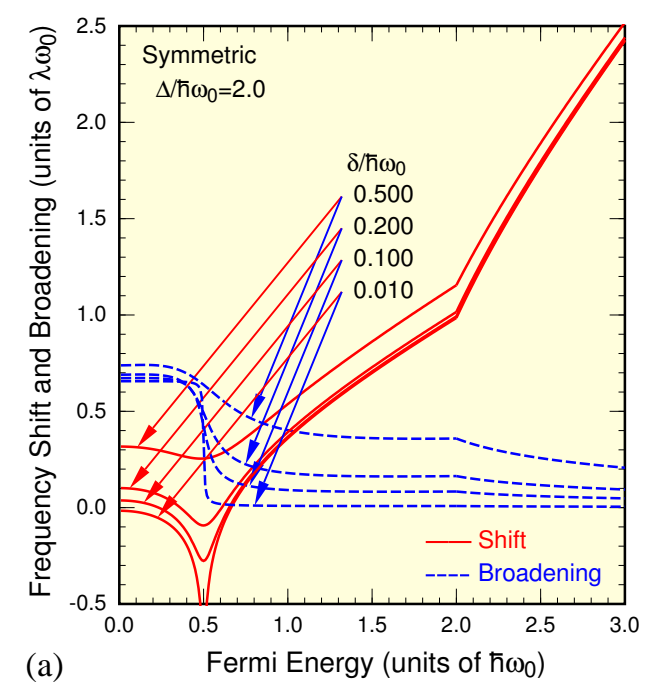

(a)

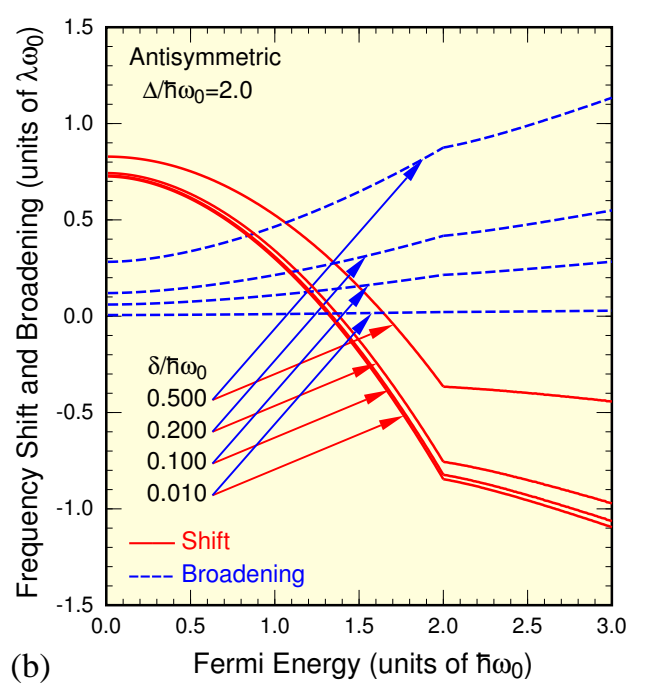

Figure 4: Some examples of calculated frequency shift and broadening of the symmetric (a) and antisymmetric mode (b) at the $\Gamma$ point in a bilayer graphene. $\Delta / \hbar \omega_{0}=2$. The amount of the broadening due to disorder is denoted by $\delta$.

where $\alpha$ represents $\Gamma$ or $K$ and we have neglected the phonon occupation due to large $\omega_{\alpha}$ at room temperature. This simply shows that the electron lifetime is inversely proportional to the coupling parameter $\lambda_{\alpha}$ and to the density of states at the energy of the final state. The phonon emission is possible only when the energy of the initial electron is larger than that of the phonon to be emitted. Otherwise, the final states are fully occupied at zero temperature and the phonon emission never takes place. In this sense, the zone-boundary phonon has another advantage over the zone-center phonon. Therefore, the zone-boundary phonon gives dominant scattering for high-field transport in graphene and in nanotube owing to its smaller frequency, larger coupling constant, and large backward scattering.

\section{Bilayer Graphene}

We consider a bilayer graphene which is arranged in the $\mathrm{AB}$ (Bernal) stacking. The upper layer is denoted as 1 and the lower

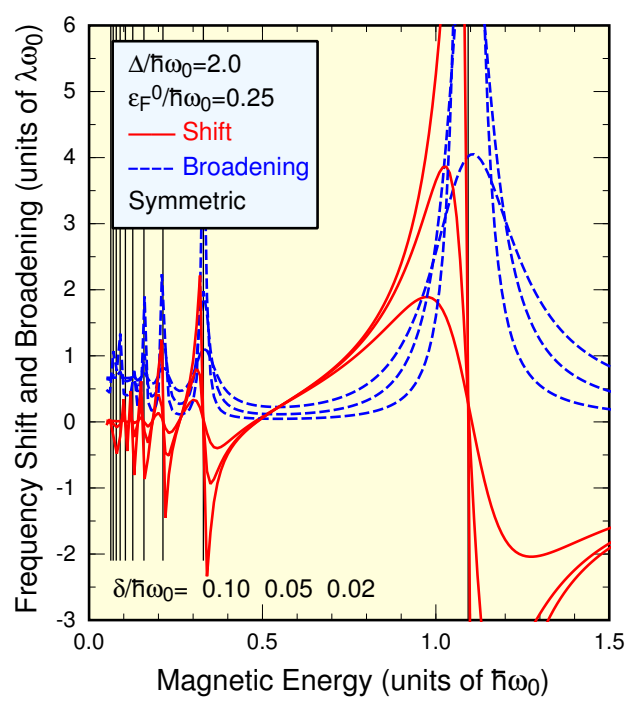

Figure 5: Calculated frequency shift and broadening of the symmetric mode in a bilayer graphene as a function of the effective magnetic energy $\hbar \omega_{\mathrm{c}}$. The thin vertical straight lines denote the field where the energy difference of optically allowed Landau levels become equal to the phonon energy. $\Delta / \hbar \omega_{0}=2$. $\varepsilon_{\mathrm{F}}^{0} / \hbar \omega_{0}=0.25$.

layer denoted as 2 . In each layer, the unit cell contains two carbon atoms denoted by $A_{1}$ and $B_{1}$ in layer 1 and $A_{2}$ and $B_{2}$ in layer 2. For the inter-layer coupling, we include only the coupling between vertically neighboring atoms. Then, electronic states are described by the k·p Hamiltonian [34, 35]:

$$
\mathcal{H}=\left(\begin{array}{cccc}
\mathrm{A}_{1} & \mathrm{~B}_{1} & \mathrm{~A}_{2} & \mathrm{~B}_{2} \\
\gamma & \gamma \hat{k}_{-} & 0 & 0 \\
\gamma \hat{k}_{+} & 0 & \Delta & 0 \\
0 & \Delta & 0 & \gamma \hat{k}_{-} \\
0 & 0 & \gamma \hat{k}_{+} & 0
\end{array}\right),
$$

where $\Delta(\approx 0.4 \mathrm{eV})$ represents the inter-layer coupling between sites $\mathrm{B}_{1}$ and $\mathrm{A}_{2}$.

Let us define

$$
\varepsilon(k)=\sqrt{\left(\frac{\Delta}{2}\right)^{2}+(\gamma k)^{2}}, \quad \gamma k=\varepsilon(k) \sin \psi, \quad \frac{\Delta}{2}=\varepsilon(k) \cos \psi,
$$

where $\psi$ vanishes for $k=0$ and approaches $\pi / 2$ with increasing $k$. Then, the eigen energies are given by

$$
\varepsilon_{ \pm 1}(k)= \pm 2 \varepsilon(k) \sin ^{2}(\psi / 2), \quad \varepsilon_{ \pm 2}(k)= \pm 2 \varepsilon(k) \cos ^{2}(\psi / 2)
$$

The band $\varepsilon_{+1}(k)$ represents the lowest conduction band which touches the highest valence band $\varepsilon_{-1}(k)$ at $k=0$. The bands $\varepsilon_{ \pm 2}(k)$ are the excited conduction and valence bands and $\varepsilon_{+2}(k)-\varepsilon_{+1}(k)=\delta$ independent of $k$. In the vicinity of $\varepsilon=0$, the Hamiltonian is reduced to a $(2,2)$ form for the basis set $\left(A_{1}, B_{2}\right)$ and the energy dispersion becomes $\varepsilon(k)= \pm \hbar^{2} k^{2} / 2 m^{*}$ with $m^{*}=\hbar^{2} \Delta / 2 \gamma^{2}$. The Landau levels become $\pm \hbar \omega_{\mathrm{c}} \sqrt{n(n+1)}$ $(n=0, \pm 1, \cdots)$ with $\omega_{\mathrm{c}}=e B / m^{*} c$.

In bilayer graphene, optical phonons are classified into symmetric and antisymmetric modes in which the displacement of 


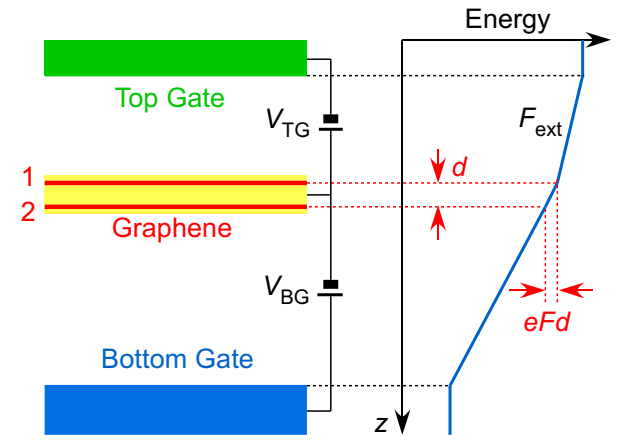

Figure 6: A schematic illustration of the bilayer graphene with a top gate and a bottom gate and the potential energy diagram. The distance between the layers is given by $d$, the potential difference by $e F d$, and $F_{\text {ext }}$ represents the field due to the top gate.

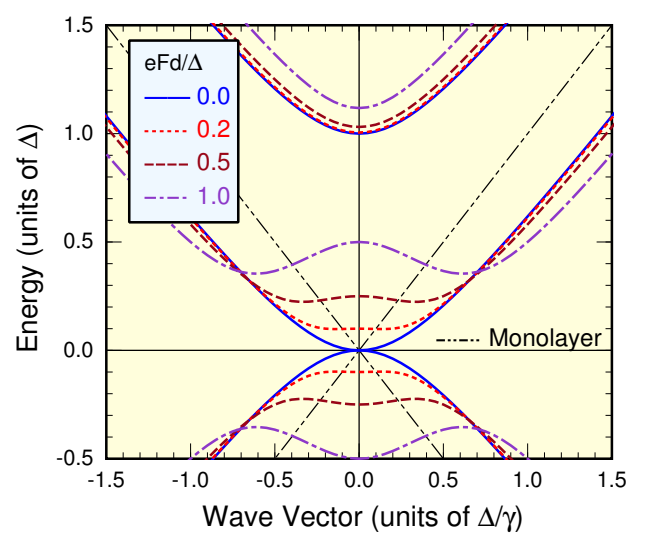

Figure 7: The energy dispersion for varying values of the potential difference $e F d$. The dot-dot-dashed lines show that of a monolayer graphene.

the top and bottom layers are in-phase and out-of-phase, respectively. They are affected by electron-phonon interactions in a different manner [28]. The symmetric mode causes interband transitions between $\varepsilon_{ \pm 1}(k)$ and therefore exhibits logarithmic singularity in a manner same as monolayer graphene when $\varepsilon_{\mathrm{F}}=\hbar \omega_{0} / 2$. On the other hand, this transition is not allowed for the antisymmetric mode but interband transitions between $\varepsilon_{+1}(k)$ and $\varepsilon_{+2}(k)$ contribute to the phonon renormalization. Figure 4 shows calculated frequency shift and broadening for two phonon modes. Figure 5 shows an example of results in the presence of a magnetic field.

One important feature is that the band structure can be strongly modified due to opening-up of a band gap by applied electric field [36]. Figure 6 shows a schematic illustration of the device structure, where $e F d$ represents the potential difference between layers 1 and 2 ( $F$ is the effective electric field and $d=0.334 \mathrm{~nm}$ is the interlayer distance). The effective Hamiltonian becomes

$$
\mathcal{H}=\left(\begin{array}{cccc}
e F d / 2 & \gamma \hat{k}_{-} & 0 & 0 \\
\gamma \hat{k}_{+} & e F d / 2 & \Delta & 0 \\
0 & \Delta & -e F d / 2 & \gamma \hat{k}_{-} \\
0 & 0 & \gamma \hat{k}_{+} & -e F d / 2
\end{array}\right)
$$

Figure 7 shows the energy bands obtained by the diagonaliza-

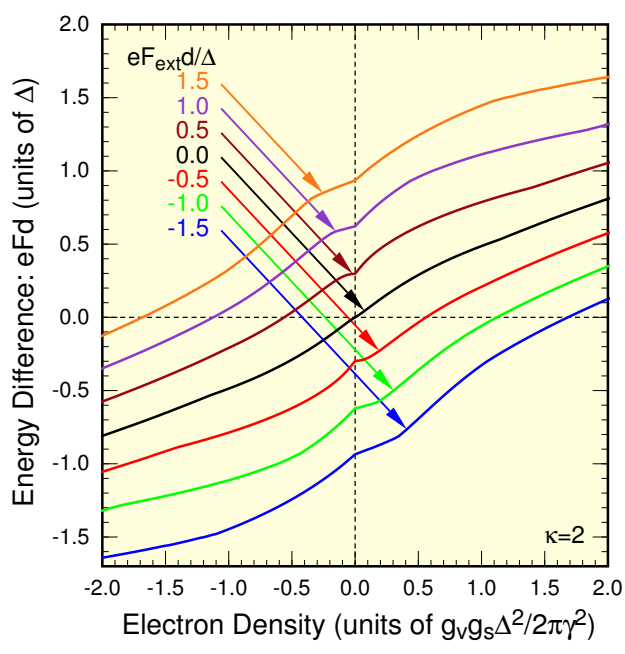

Figure 8: Calculated energy difference $e F d$ as a function of the electron concentration for various values of $e F_{\text {ext }} d$. The static dielectric constant of the environment is chosen as $\kappa=2$.

tion of this Hamiltonian. The band gap appears and the minimum gap is located at a nonzero value of $k$ depending on the field.

The effective field is determined in a self-consistent manner [36] because the electron density distribution between two layers becomes different, giving rise to interlayer potential difference. Some examples of the results of such calculations within this model Hamiltonian are shown in Fig. 8 [37]. The unit $n_{\mathrm{s}}^{0}=g_{\mathrm{v}} g_{\mathrm{s}} \Delta^{2} / 2 \pi \gamma^{2}$ is the electron concentration at $\varepsilon_{\mathrm{F}}=\Delta$ for $e F d=0$. We have $n_{\mathrm{s}}^{0} \approx 2.5 \times 10^{13} \mathrm{~cm}^{-2}$ for $\Delta \approx 0.4 \mathrm{eV}$. We can see $e F d$ can become as large as $\sim \Delta / 2$ although being dependent on $e F_{\text {ext }} d$.

Figure 9(a) shows the frequency shift (top panel), broadening (middle panel), and the spectral intensity of the symmetric component (bottom panel) as a function of the electron concentration for $e F_{\text {ext }} d=0$ [38]. We have assumed $\Delta / \hbar \omega_{0}=2$ corresponding to $\Delta \approx 0.4 \mathrm{eV}$ and $\hbar \omega_{0} \approx 0.2 \mathrm{eV}$ and $\delta / \hbar \omega_{0}=0.1$. The symmetric component shown in the figure describes the relative intensity of the Raman scattering. At $n_{\mathrm{s}}=0$ with $e F d=0$, the optical phonons are exactly classified into symmetric and antisymmetric modes. With the increase of $n_{\mathrm{s}}$, they become mixed with each other, which becomes particularly important when they cross each other. Figure 9(b) shows results for $e F_{\text {ext }} d / \hbar \omega_{0}=0.5$, for which the asymmetry is enhanced in the electron side and reduced in the hole side.

The appearance of two peaks at sufficiently high electron concentration, where the potential asymmetry is significant, is in agreement with the feature of recent experiments showing double peaks in a highly doped bilayer graphene [39]. In order to make detailed comparison with experiments, we may have to consider a small frequency splitting of symmetric and antisymmetric modes, which can be present even in the symmetric bilayer and is independent of the change in the electron concentration. 

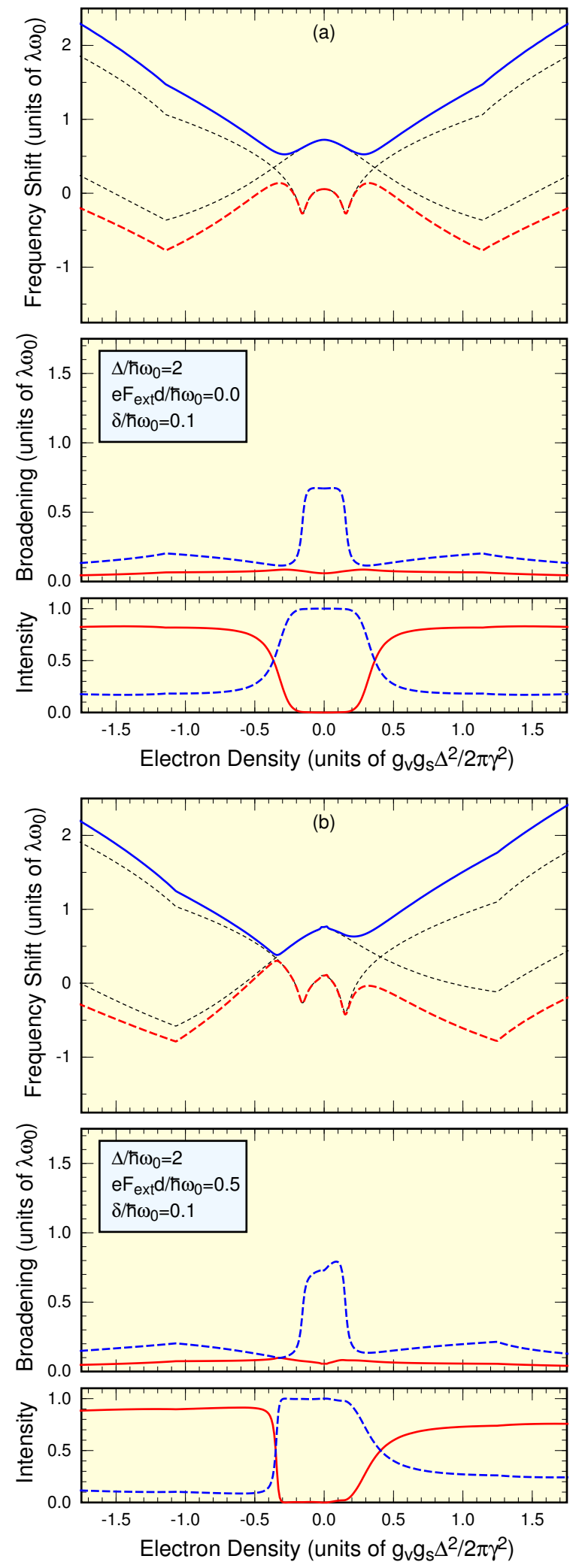

Figure 9: Calculated frequency shift, broadening, and strength of the symmetric component. The solid and dashed lines denote the high- and low-frequency modes, respectively. The thin dotted lines in the top panel show the frequencies for symmetric and antisymmetric modes calculated without inclusion of their mixing. $\Delta / \hbar \omega_{0}=2 . \delta / \hbar \omega_{0}=0.1$. (a) $e F_{\text {ext }} d / \hbar \omega_{0}=0$ and (b) 0.5 .

\section{Acknowledgments}

The author acknowledges the collaboration with Drs. H. Suzuura and M. Koshino. This work was supported in part by Grant-in-Aid for Scientific Research on Priority Area "Carbon Nanotube Nanoelectronics," by Grant-in-Aid for Scientific Research, and by Global Center of Excellence Program at Tokyo Tech "Nanoscience and Quantum Physics" from Ministry of Education, Culture, Sports, Science and Technology Japan.

\section{References}

[1] K. S. Novoselov, A. K. Geim, S. V. Morozov, D. Jiang, Y. Zhang, S. V. Dubonos, I. V. Grigorieva, and A. A. Firsov, Science 306, 666 (2004).

[2] K. S. Novoselov, A. K. Geim, S. V. Morozov, D. Jiang, M. I. Katsnelson, I. V. Grigorieva, S. V. Dubonos, and A. A. Firsov, Nature 438, 197 (2005).

[3] Y. Zhang, Y.-W. Tan, H. L. Stormer, and P. Kim, Nature 438, 201 (2005).

[4] T. Ando, Physica E 40, 213 (2007).

[5] A. H. Castro Neto, F. Guinea, N. M. Peres, K. S. Novoselov, and A. K. Geim, Rev. Mod. Phys. 81, 109 (2009).

[6] T. Ando, J. Phys. Soc. Jpn. 74, 777 (2005) and references cited therein.

[7] P. R. Wallace, Phys. Rev. 71, 622 (1947).

[8] G. S. Painter and D. E. Ellis, Phys. Rev. B 1, 4747 (1970).

[9] J. W. McClure, Phys. Rev. 104, 666 (1956).

[10] J. C. Slonczewski and P. R. Weiss, Phys. Rev. 109, 272 (1958).

[11] D. P. DiVincenzo and E. J. Mele, Phys. Rev. B 29, 1685 (1984).

[12] G. W. Semenoff, Phys. Rev. Lett. 53, 2449 (1984).

[13] H. Ajiki and T. Ando, J. Phys. Soc. Jpn. 62, 1255 (1993).

[14] C. L. Kane and E. J. Mele, Phys. Rev. Lett. 78, 1932 (1997).

[15] A. C. Ferrari, J. C. Meyer, V. Scardaci, C. Casiraghi, M. Lazzeri, F. Mauri, S. Piscanec, D. Jiang, K. S. Novoselov, S. Roth, and A. K. Geim, Phys. Rev. Lett. 97, 187401 (2006).

[16] A. Gupta, G. Chen, P. Joshi, S. Tadigadapa, and P.C. Eklund, Nano Lett. 6, 2667 (2006).

[17] K. Ishikawa and T. Ando, J. Phys. Soc. Jpn. 75, 084713 (2006).

[18] T. Ando, J. Phys. Soc. Jpn. 75, 124701 (2006).

[19] T. Ando, J. Phys. Soc. Jpn. 76, 024712 (2007).

[20] T. Ando, J. Phys. Soc. Jpn. 77, 014707 (2008).

[21] See for example, W. A. Harrison, Electronic Structure and the Properties of Solids (W.H. Freeman and Company, San Francisco, 1980).

[22] N. A. Viet, H. Ajiki, and T. Ando, J. Phys. Soc. Jpn. 63, 3036 (1994).

[23] M. Lazzeri and F. Mauri, Phys. Rev. Lett. 97, 266407 (2006).

[24] S. Pisana, M. Lazzeri, C. Casiraghi, K. S. Novoselov, A. K. Geim, A. C. Ferrari, and F. Mauri, Nat. Mat. 6, 198 (2007).

[25] J. Yan, Y. Zhang, P. Kim, and A. Pinczuk, Phys. Rev. Lett. 98, 166802 (2007).

[26] M. O. Goerbig, J.-N. Fuchs, K. Kechedzhi, and V. I. Falko, Phys. Rev. Lett. 99, 087402 (2007).

[27] C. Faugeras, M. Amado, P. Kossacki, M. Orlita, M. Sprinkle, C. Berger, W. A. de Heer, and M. Potemski, Phys. Rev. Lett. 103, 186803 (2009).

[28] T. Ando, J. Phys. Soc. Jpn. 76, 104711 (2007).

[29] H. Farhat, H. Son, Ge. G Samsonidze, S. Reich, M. S. Dresselhaus, and J. Kong, Phys. Rev. Lett. 99, 145506 (2007).

[30] Y. Wu, J. Maultzsch, E. Knoesel, B. Chandra, M. Huang, M. Y. Sfeir, L. E. Brus, J. Hone, and T. F. Heinz, Phys. Rev. Lett. 99, 027402 (2007).

[31] K. Sasaki, R. Saito, G. Dresselhaus, M. S. Dresselhaus, H. Farhat, and J. Kong, Phys. Rev. B 78, 235405 (2008).

[32] J. S. Park, K. Sasaki, R. Saito, W. Izumida, M. Kalbac, H. Farhat, G. Dresselhaus, and M. S. Dresselhaus, Phys. Rev. B 80, 081402 (2009).

[33] H. Suzuura and T. Ando, J. Phys. Soc. Jpn. 77, 044703 (2008).

[34] E. McCann and V. I. Falko, Phys. Rev. Lett. 96, 086805 (2006).

[35] M. Koshino and T. Ando, Phys. Rev. B 73, 245403 (2006).

[36] E. McCann, Phys. Rev. B 74, 161403 (2006).

[37] T. Ando and M. Koshino, J. Phys. Soc. Jpn. 78, 104716 (2009).

[38] T. Ando and M. Koshino, J. Phys. Soc. Jpn. 78, 034709 (2009).

[39] L. M. Malard, D. C. Elias, E. S. Alves, and M. A. Pimenta, Phys. Rev. Lett. 101, 257401 (2008). 